\title{
Extreme THz nonlinearities in bulk and nanostructured semiconductors
}

\author{
A. Sell ${ }^{1}$, A. A. Anappara ${ }^{1}$, T. Kampfrath ${ }^{2}$, K. v. Volkmann ${ }^{2}$, M. Wolf ${ }^{2}$, J.T. Steiner ${ }^{3}$, M. Kira ${ }^{3}$, \\ S.W. Koch ${ }^{3}$, G. Biasiol ${ }^{4}$, L. Sorba ${ }^{4,5}$, A. Tredicucci ${ }^{5}$, A. Leitenstorfer ${ }^{1}$, and R. Huber ${ }^{1}$ \\ ${ }^{1}$ Department of Physics and Center for Applied Photonics, \\ University of Konstanz, 78464 Konstanz, Germany. \\ ${ }^{2}$ Department of Physics, Freie Universität Berlin, 14195 Berlin, Germany. \\ ${ }^{3}$ Department of Physics, Universität Marburg, 35032 Marburg, Germany. \\ ${ }^{4}$ Laboratorio Nazionale TASC CNR-INFM, 34012 Trieste, Italy. \\ ${ }^{5}$ Laboratorio NEST, CNR-INFM and Scuola Normale Superiore, 56127 Pisa, Italy.
}

\begin{abstract}
Phase-locked electromagnetic transients in the terahertz $(\mathrm{THz})$ spectral domain have become a unique contact-free probe of the femtosecond dynamics of low-energy excitations in semiconductors. Access to their nonlinear response, however, has been limited by a shortage of sufficiently intense $\mathrm{THz}$ emitters. Here we introduce a novel high-field source for $\mathrm{THz}$ transients featuring peak amplitudes of up to $108 \mathrm{MV} / \mathrm{cm}$. This facility allows us to explore the non-perturbative response of semiconductors to intense fields tailored with sub-cycle precision. In a first experiment intense transients drive Rabi-oscillations between excitonic states in $\mathrm{Cu}_{2} \mathrm{O}$, implying exciting perspectives for future $\mathrm{THz}$ quantum optics. At electric fields beyond $10 \mathrm{MV} / \mathrm{cm}$, we observe the breakdown of the power expansion of the nonlinear polarization in bulk semiconductors. Furthermore, we employ the intense magnetic field components of our transients to coherently control spin waves in antiferromagnetically ordered solids. Finally, intersubband cavity polaritons in semiconductor microcavities are exploited to push light-matter coupling to an unprecedented ultrastrong and sub-cycle regime.
\end{abstract}

Keywords: High field terahertz; Coherent control; Extreme nonlinearities; Field resolved measurements

\section{INTRODUCTION}

Few-cycle light pulses in the elusive $\mathrm{THz}$ region of the electromagnetic spectrum have enabled new directions in fundamental science and technology ${ }^{1}$. This is mainly due to two unique benefits of $\mathrm{THz}$ optoelectronics: The possibility of generating inherently phase stable transients and the availability of field-sensitive detectors. Recently these technologies have been extended across an ultrabroad spectral window approaching the near infrared ${ }^{2-5}$. Thus, they are ideally applicable to resonant probing of low-energy elementary excitations in condensed matter on a sub-cycle time scale $^{6-11}$. Actually, novel applications which have been envisioned for $\mathrm{THz}$ nonlinear optics and coherent control schemes, require enhanced field amplitudes ${ }^{12-15}$. Until recently this domain has been reserved to large-scale facilities such as synchrotrons and free-electron lasers.

Here we introduce a hybrid table-top laser system, which combines a flexible and compact Er:fiber laser with a high energy Ti:sapphire amplifier to provide unprecedented field amplitudes while maintaining the benefits of $\mathrm{THz}$ optoelectronics. Our setup generates few-cycle electromagnetic transients with inherently stable carrier-envelope phase (CEP) and center frequencies tunable from 1 to $107 \mathrm{THz}$. Peak electric fields of up to $108 \mathrm{MV} / \mathrm{cm}$ with maximum powers of $10^{8} \mathrm{~W}$ and energies as large as $19 \mu \mathrm{J}$ pave the way to extreme nonlinear THz optics ${ }^{16}$. In the following sections we discuss this new laser system and four lead-off examples of extreme nonlinearities driven by the electric and magnetic fields of the transients, which go beyond the perturbative regime.

Ultrafast Phenomena in Semiconductors and Nanostructure Materials XIV, edited by Jin-Joo Song, Kong-Thon Tsen, Markus Betz, Abdulhakem Y. Elezzabi, Proc. of SPIE Vol. 7600, 76001S · C 2010 SPIE' CCC code: 0277-786X/10/\$18 · doi: 10.1117/12.840959 


\section{NOVEL SOURCE OF HIGH FIELD THZ TRANSIENTS}

Fig. 1 displays a schematic of our THz source. A low-noise four-branch Er:fiber system with all-fiber frequency conversion stages serves as a starting point. The output pulses from the first arm at a center wavelength of $1.55 \mu \mathrm{m}$ is frequency doubled and seeded into a Ti:sapphire amplifier encompassing a regenerative cavity and a double-pass power stage (repetition rate: $1 \mathrm{kHz}$ ). A train of 5-mJ pulses of a duration of $100 \mathrm{fs}$ featuring rms power fluctuations well below $0.2 \%$ (integrated over $30 \mathrm{~s}$ ) is obtained. Three schemes for the generation of intense and widely tunable $\mathrm{THz}$ fields from this pump as well as from the various fiber branches are implemented:

(i) Optical rectification of the 5-mJ pulses in a large-area ZnTe crystal provides transients with center frequencies around $1 \mathrm{THz}$. The single-cycle wave forms reach peak electric field amplitudes of up to $400 \mathrm{kV} / \mathrm{cm}$ and cover the lowfrequency range from 0.1 to $3 \mathrm{THz}$.

(ii) In a second approach we obtain even more intense $\mathrm{THz}$ pulses. Two identical two-stage optical parametric amplifiers (OPAs) are pumped with the Ti:sapphire output pulses. A white-light continuum generated in a sapphire window serves as a shared seed for both amplifiers and ensures a high degree of mutual phase coherence. The signal components are superimposed for type-II or type-I difference frequency generation (DFG) in a GaSe or $\mathrm{AgGaS}_{2}$ crystal. This way, we achieve pulse energies as large as $19 \mu \mathrm{J}$. Since both OPAs share the same frequency comb as a seed, the carrier-envelope offset is expected to cancel in the DFG process (see next section and Refs. 17 and 18). The center frequencies are tunable up to $72 \mathrm{THz}^{16}$.

(iii) Finally, we generate phase-stable THz transients with record-high center frequencies of up to $107 \mathrm{THz}$ directly in the fiber laser. One amplifier branch is frequency shifted to generate tunable spectra in the wavelength range from 1.0 to $1.4 \mu \mathrm{m}$. For this purpose we employ dispersion-optimized highly nonlinear bulk germanosilicate fibers, which guarantee excellent stability. A second branch is operated at the fundamental wavelength of $1.55 \mu \mathrm{m}$. Both pulse trains are superimposed and focussed into a periodically poled $\mathrm{LiNbO}_{3}$ crystal to generate $\mathrm{THz}$ transients via $\mathrm{DFG}^{19}$. The mutual phase jitter of both branches amounts to 43 attoseconds, which is less than one percent of the oscillation period of the carrier wave ${ }^{20}$. Therefore, CEP stability of the THz-pulses is expected also for this scheme. Due to the high repetition rate of $49 \mathrm{MHz}$ and an average $\mathrm{THz}$ power of up to $1.4 \mathrm{~mW}$, this mid- to near-infrared source is ideally suited for sensitive spectroscopy with field sensitive detection. In a later stage, the pulses may be further amplified in Ti:sapphire pumped parametric optics, similar to Ref. 21.

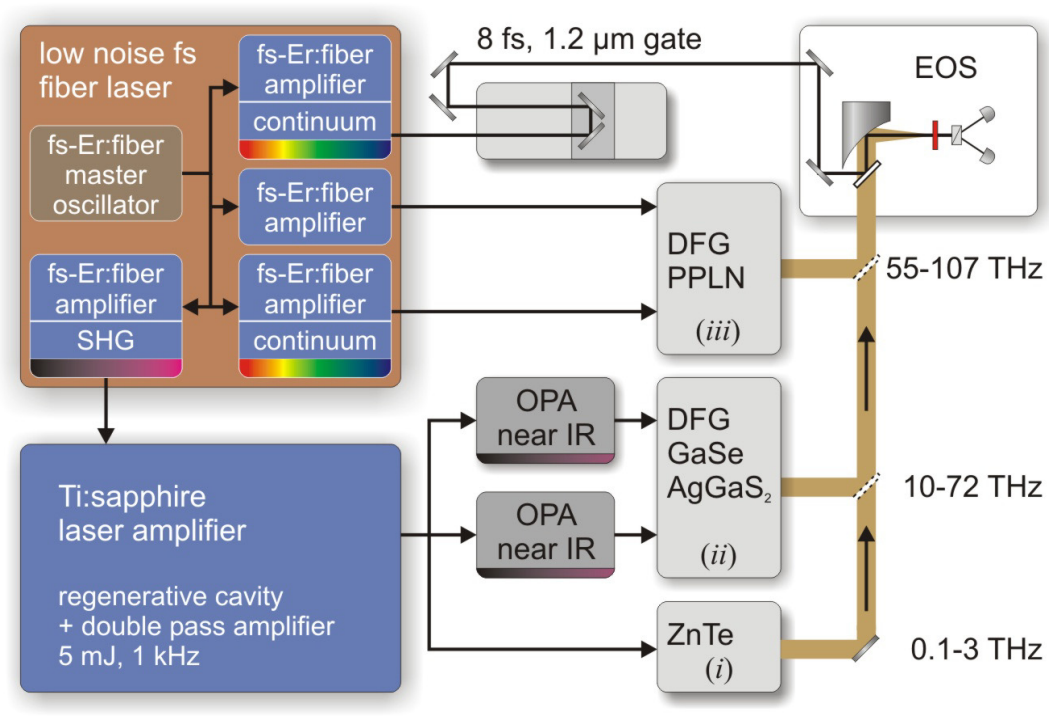

Figure 1. Schematic setup of the novel hybrid laser system with a four branch Er:fiber laser, a high-power Ti:sapphire amplifier, optical parametrical amplifiers (OPA), difference frequency stages (DFG), and a setup for electro-optic sampling (EOS). The accessible frequency ranges are indicated next to the THz-generation stages. 


\section{ULTRABROADBAND ELECTRO-OPTIC SAMPLING}

The electric field traces of the $\mathrm{THz}$ transients from all three generation schemes are detected via electro-optic sampling with the pulses of a fourth Er:fiber branch. To this end, the 100-fs pulses from the Er:Fiber amplifier are coupled into a robust splice assembly of two nonlinear bulk fibers. The dispersion profiles of these fibers are numerically optimized for extremely broadband output spectra. Pulses as short as $7.8 \mathrm{fs}$ with smooth spectra spanning the range from $0.9 \mu \mathrm{m}$ to $1.4 \mu \mathrm{m}$ are available after this compression stage $\mathrm{e}^{22}$. Recently, we demonstrated even shorter pulses comprising only a single oscillation cycle of the carrier wave with a duration of $4.3 \mathrm{fs}$ by coherently combining two such ultrabroadband spectra $^{23}$. Pulses of this kind permit electro-optical gating even at the highest center frequency. The bandwidth is sufficient to resolve oscillation frequencies far above $100 \mathrm{THz}$ directly in the time domain ${ }^{5}$.
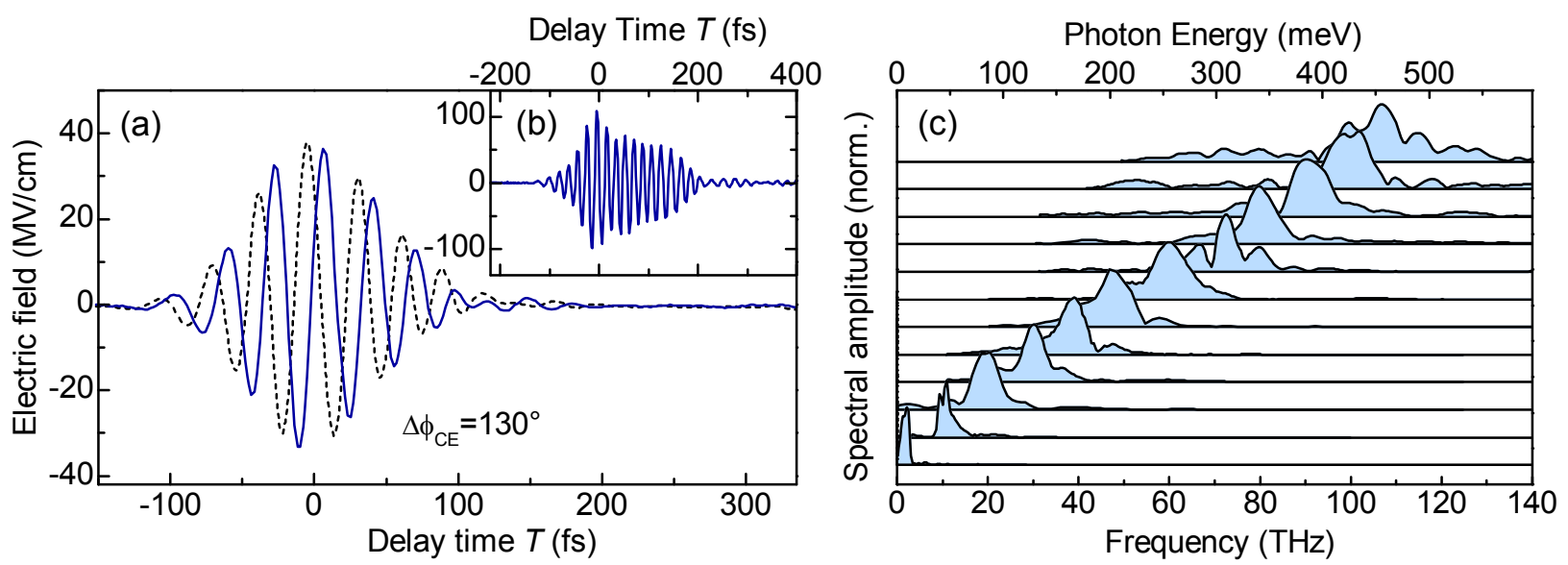

Figure 2. (a) Typical THz transient obtained from type-II difference frequency mixing in GaSe (blue line). The carrier envelope phase $\phi_{\mathrm{CE}}$ is changed via the temporal overlap of the near infrared pump waves (dashed line). (b) THz transient from a thick emitter crystal, which reaches a peak field of $108 \mathrm{MV} / \mathrm{cm}$. (c) Output spectra of the new hybrid laser source, obtained as Fourier transforms of the transients. The tuning range includes center frequencies from $1 \mathrm{THz}$ up to 107 THz.

Fig. 2 (a) depicts the temporal trace of a typical high-field transient generated from scheme (ii). The fact, that it is possible to scan the field shape in a stroboscopic manner, is the ultimate proof of the phase stability of the OPA-based difference frequency technique. Moreover, by simply varying the temporal overlap of both pump pulses, the carrier envelope phase might be freely selected, as illustrated by the dashed curve. A proper selection of the generation crystal provides peak fields of up to $108 \mathrm{MV} / \mathrm{cm}$, as shown in Fig. 2 (b). This value is easily calibrated by means of a thermal powermeter, since the average $\mathrm{THz}$ power reaches a value of $19 \mathrm{~mW}$. These transients are by far the most intense $\mathrm{THz}$ pulses detected electro-optically to date ${ }^{16}$.

Fourier transformation of transients from all three generation schemes yield phase and amplitude spectra which highlight the unprecedented bandwidth of more than 10 optical octaves with frequency components extending from $100 \mathrm{GHz}$ to $140 \mathrm{THz}$ [Fig. 2 (c)]. With photon energies spanning the entire range from $0.4 \mathrm{meV}$ to $580 \mathrm{meV}$, many important resonances in condensed matter are directly accessible by intense $\mathrm{THz}$ pulses with full amplitude and phase resolution.

\section{NON-PERTURBATIVE THZ NONLINEARITIES IN CONDENSED MATTER}

Our transients reach electric fields as high as $1 \mathrm{~V} / \AA$ which is comparable to the potential gradients within typical atoms. Therefore we expect to induce an extremely nonlinear response when applying these pulses to condensed matter. Electro-optic sampling provides direct access to amplitude and absolute phase information of the reemitted fields. In the following, we discuss four lead-off examples of non-perturbative nonlinearities in solids. 


\subsection{Coherent Control of Intra-Excitonic Transitions}
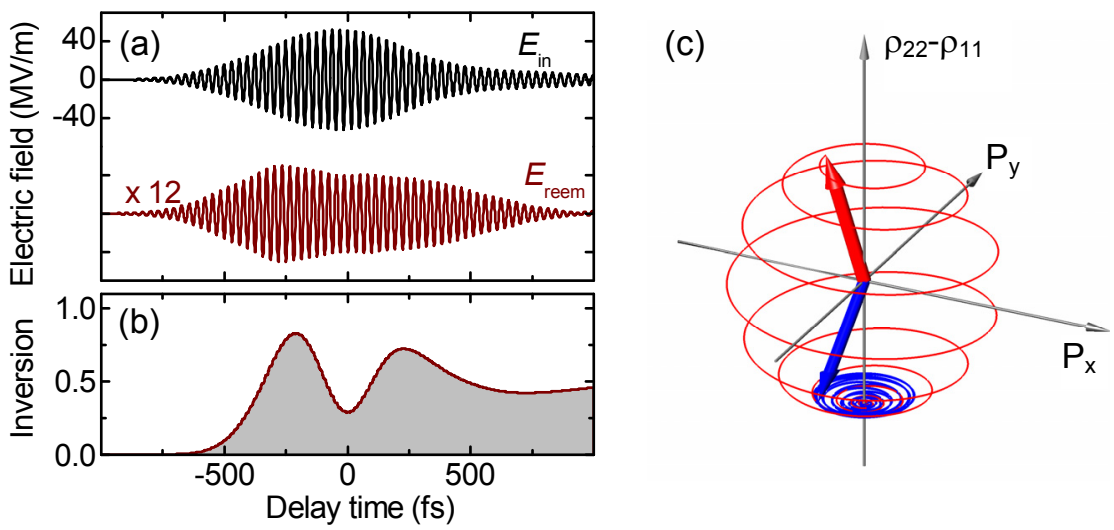

Figure 3. (a) $\mathrm{THz}$ transients resonantly drive the internal $1 \mathrm{~s}-2 \mathrm{p}$ transition of paraexcitons in $\mathrm{Cu}_{2} \mathrm{O}$ (black curve). The system reemits a field (red curve), which exhibits a non-monotonous envelope characteristic of up to two internal Rabi cycles. (b) Results of a microscopic theory confirm an oscillatory change of the population inversion of the $1 \mathrm{~s}-2 \mathrm{p}$ two-level system. (c) Bloch sphere representation of the 1s-2p intra-excitonic two-level system.

$\mathrm{THz}$ transients are especially well suited to couple to low-energy excitations of quasiparticles which may not even be accessible by other means for reasons such as vanishing interband dipole matrix elements. Electron-hole pairs in semiconductors which are bound by Coulomb-interactions, are an important example. They exhibit a level structure which is analogous to the hydrogen atom and have been discussed as potential candidates for Bose Einstein condensation (BEC) in solid-state systems.

Excitons that are relevant for BEC are usually long-lived and often exhibit weak if any coupling to visible and nearinfrared light. Thus, it is a fundamental challenge to control these excitations by conventional optical techniques. The optically dark 1s paraexcitons in $\mathrm{Cu}_{2} \mathrm{O}$ represent a prototypic example. Intense $\mathrm{THz}$ radiation turns out to be an elegant way not only to visualize these quasiparticles, but also to control their orbital degree of freedom: Multi-terahertz fields of the order of $0.4 \mathrm{MV} / \mathrm{cm}$ are used to promote $1 \mathrm{~s}$ paraexcitons in $\mathrm{Cu}_{2} \mathrm{O}$ into the $2 \mathrm{p}$ state, coherently. Fig. 3 (a) depicts an example of the nonlinear field response monitored by ultrabroadband electro-optic sampling. We identify up to two internal Rabi cycles, which is in quantitative agreement with a microscopic many-body theory ${ }^{24,25}$. The results point out a promising route for preparing ultracold exciton gases which may be eligible for potential Bose-Einstein condensation in semiconductors.

\subsection{Extreme THz-nonlinearities in bulk semiconductors}
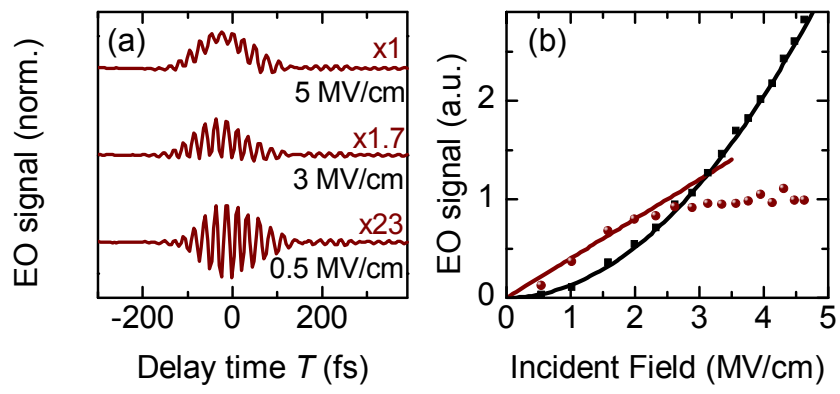

Figure 4. (a) Extremely nonlinear electro-optic sampling of transients with increasing peak fields in a 90- $\mu$ m-thick GaSe detector crystal. The peak field is indicated next to each transient. (b) Field dependence of the unipolar (squares) and the rapidly oscillating components (circles) in (a). 
In a second example, we investigate higher-order nonlinearities in bulk semiconductors. To this end, we increase the $\mathrm{THz}$ power yet further to reach peak intensities of $15 \mathrm{TW} / \mathrm{cm}^{2}$ (see Ref. 16). The induced effects may be directly observed in the electro-optic sensor itself (see Fig. 4): Up to fields of $2 \mathrm{MV} / \mathrm{cm}$, the electro-optic signal scales linearly with the THz amplitude [circles in Fig. 4(b)], as one would expect for a $\chi^{(2)}$ nonlinearity. However, for increasing THz field strengths, a unipolar component which is superimposed on the transient is clearly discernible in the time-domain data. The component which oscillates with the carrier frequency of the THz pulse saturates while the unipolar signal (squares) increases quadratically, which indicates $\chi^{(3)}$ and $\chi^{(4)}$ processes involving two and three THz photons, respectively. At intensities beyond $5 \mathrm{MV} / \mathrm{cm}$, we start to observe $\mathrm{THz}$ induced continuum generation, interband luminescence, and ultimately $\mathrm{THz}$ radiation damage of the detector, indicating a catastrophic divergence of the power series expansion of the nonlinear polarization. More detailed investigations, which also include effects of crystalline anisotropy, are under way.

\subsection{THz magnetooptics: Femtosecond coherent control of antiferromagnetic magnons}

Nonlinear optics in the THz domain up to now has solely relied on the strong coupling of the electric field component to the charge degree of freedom. The magnetic field coupling to magnetic dipoles usually is weaker by a factor given by the fine structure constant $\alpha=1 / 137$ and has been considered to be negligible. With our high-field source we explore, how intense single-cycle transients interact with magnetically ordered solids. We demonstrate for the first time, that the $\mathrm{THz}$ field couples directly to the spin degree of freedom.

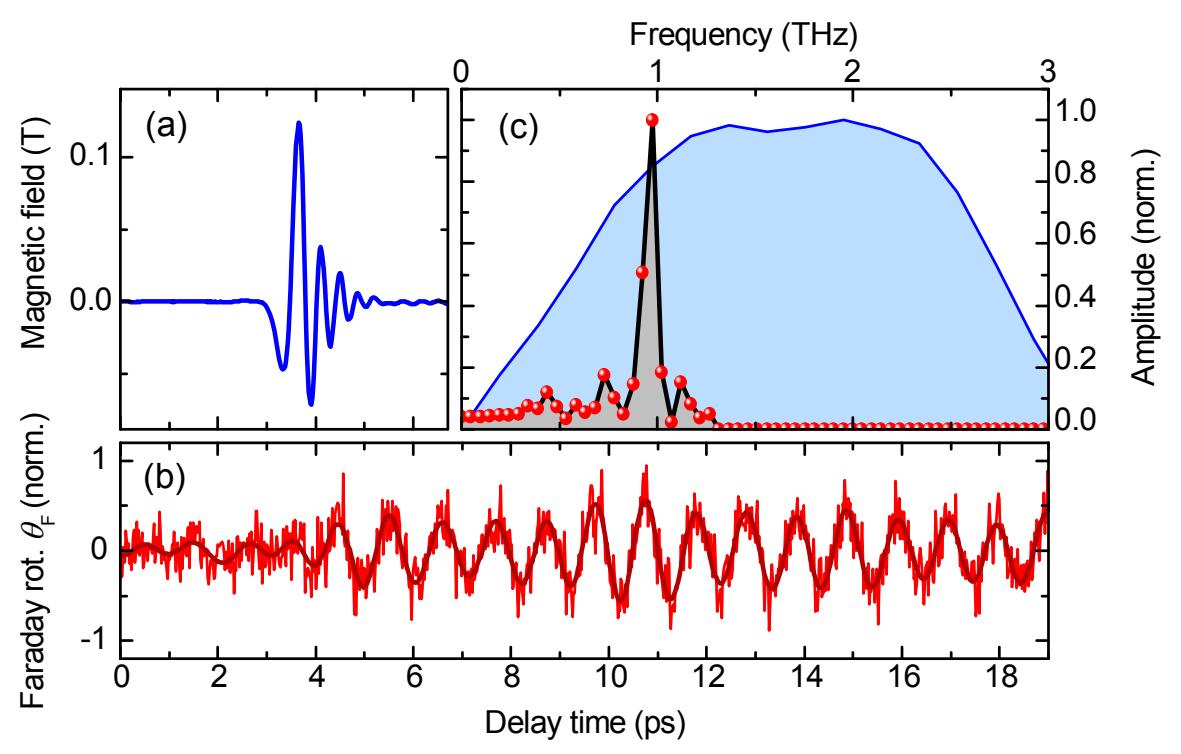

Figure 5. (a) Magnetic component of the $\mathrm{THz}$ transient incident on the $\mathrm{NiO}$ sample. (b) Faraday rotation of an 8-fs probe pulse copropagating through the antiferromagnetic sample. (c) Fourier transform of the excitation transient (blue) and the spin wave oscillations from (b).

For our experiment we choose the prototypic antiferromagnet $\mathrm{NiO}$, which we expose to single-cycle transients as depicted in Fig. 5 (a). The peak electric field of $0.4 \mathrm{MV} / \mathrm{cm}$ corresponds to a magnetic field amplitude of 0.3 Tesla. The spectrum of our excitation pulse is depicted in Fig. 5 (c) and spans a frequency interval from 0.1 to $3 \mathrm{THz}$. An 8-fs nearinfrared pulse probes any dynamics in the solid which is induced by the THz pulse. To our surprise, the strong electric field component leaves no measurable trace in the sample. Especially, there is no signature of any thermal heating of the electron-system. Instead, we observe a coherent magnon which is excited exclusively via direct magnetic Zeeman interaction of the transient with the spins. It manifests itself as a periodic Faraday-rotation of the probe pulse after the THz-excitation as depicted in Fig. 5 (b). These collective spin oscillations exhibit a frequency of $1 \mathrm{THz}$ and a long dephasing time of $39 \mathrm{ps,} \mathrm{corresponding} \mathrm{to} \mathrm{a} \mathrm{narrow} \mathrm{line} \mathrm{in} \mathrm{the} \mathrm{Fourier} \mathrm{transformation} \mathrm{[Fig.} 5$ (c)]. This is the first time resolved observation of a magnon in an antiferromagnet with vanishing net-magnetisation. In a further step a phase- 
locked sequence of $\mathrm{THz}$ magnetic transients allows us to coherently switch the magnon oscillation on and off, on a single-cycle time scale (not shown here, see Ref. 26). Our experiment opens up a new route to directly access the spins in essentially all kinds of magnetically ordered solids. It may also inspire novel applications in next-generation high-speed memory devices and spintronics.

Beyond applications in semiconductors, we are currently also exploring the utility of our high-field $\mathrm{THz}$ facility in nonlinear optics of molecules and atoms. Due to the large ponderomotive potential, strong $\mathrm{THz}$ fields interacting with atoms may in principle be an attractive option for the generation of extremely high-order harmonics.

\subsection{Ultrastrong light-matter coupling: Switching on a sub-cycle timescale}

A fundamentally different class of $\mathrm{THz}$ nonlinear optics is addressed in the final set of experiments: ultrastrong lightmatter coupling with the vacuum field of a microcavity rather than with an intense coherent external field. To enhance the interaction of light with electronic excitations, semiconductor microcavities spatially confine a photon field to the location of a semiconductor based light emitter. The confinement allows the electronic system to absorb and spontaneously reemit a photon repeatedly before dissipation sets in. This situation gives rise to mixed light-matter modes, so-called cavity polaritons. Recent efforts concentrate on the frontier of ultrastrong coupling, where the rate of photon exchange, i.e. the vacuum Rabi frequency, becomes comparable with the oscillation period of light itself $f^{27,28,29}$. Unconventional quantum electro-dynamics (QED) phenomena have been suggested for situations, where the interaction strength is modulated non-adiabatically ${ }^{30}$. While a well defined confinement has been achieved in all three spatial dimensions, control in the fourth dimension - time - has been barely developed.

(a)

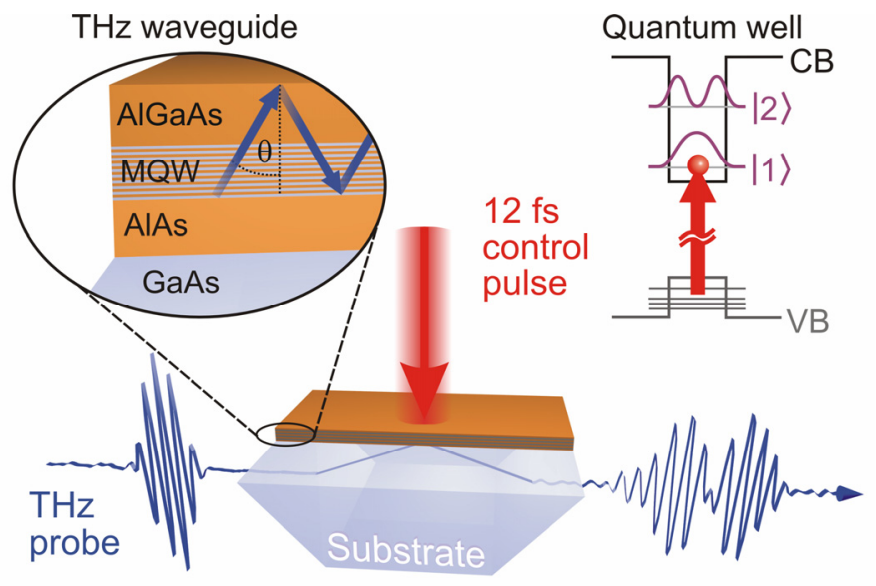

(b)

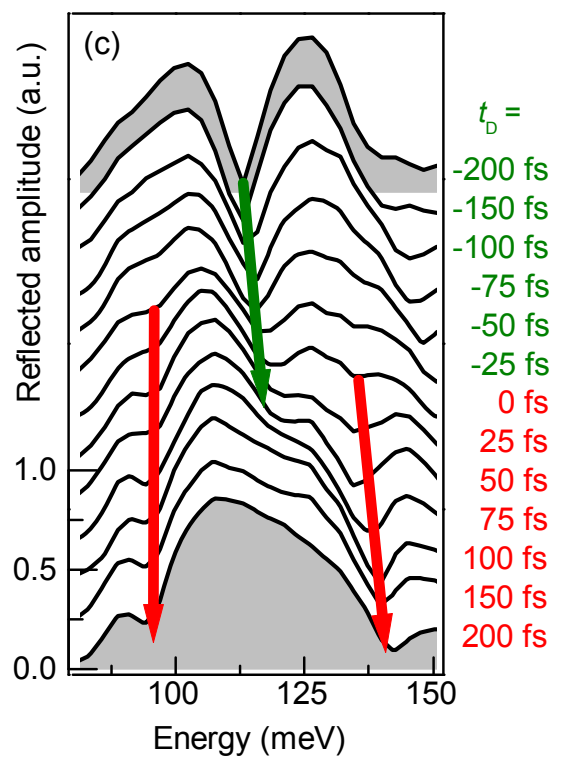

Figure 6. (a) Schematic of the femtosecond optical switch: A planar AlGaAs-waveguide based on total internal reflection encases 50 undoped GaAs quantum wells separated by AlGaAs barriers. Near infrared 12-fs control pulses populate level $|1\rangle$, as depicted in the energy level diagram in (b). The buildup of cavity polaritons is monitored by a THz probe pulse reflected off the cavity. (c) THz reflectance spectra given for various delay times $t_{\mathrm{D}}$ between the near infrared pump and the THz probe pulse. The spectra reveal the non-adiabatic switch-on dynamics of ultrastrongly coupled cavity polaritons. Green arrow: bare cavity resonance, red arrows: intersubband cavity polariton resonances.

In our experiment, we demonstrate an all-optical switch to tune from weak to ultrastrong light-matter interaction and turn on maximum coupling on the femtosecond scale ${ }^{31}$. Our resonator comprises a planar AlAs step index waveguide, containing undoped GaAs quantum wells which serve as the light emitter [Fig. 6 (a)]. Switching takes place by photoinjecting electrons into the lowest electronic subband $|1\rangle$ of the quantum wells with a 12-fs near-infrared laser pulse 
[Fig. 6 (b)]. This process activates a mid-infrared transition to the next higher subband $|2\rangle$ which may efficiently couple to TM polarized waveguide modes propagating at an angle of $65^{\circ}$. Ultrashort multi-terahertz pulses trace the formation of light-matter interaction as a function of the delay time $t_{\mathrm{D}}$ following the control pulse [Fig. $6(\mathrm{c})$ ]. Within less than a cycle of light, the system changes abruptly from a bare microcavity to an ultrastrongly coupled cavity polariton system. In this regime, a novel class of extremely non-adiabatic phenomena becomes observable: We monitor directly, by detection of the reemitted terahertz field amplitude, how a coherent photon population converts to cavity polaritons during the ultrafast switching event. This system forms a first promising laboratory for unprecedented sub-cycle QED effects and represents an efficient room-temperature switching device at the ultimate speed.

\section{CONCLUSIONS}

We have introduced a novel source of phase-locked few-cycle transients in the intriguing $\mathrm{THz}$ and mid-infrared range with peak intensities outperforming even large-scale facilities such as free-electron lasers, by several orders of magnitude. Simultaneously, the tuning range marks a new record for table-top CEP-stable THz systems. Even the highest frequency pulses are gated electro-optically giving direct access to the temporal trace of the oscillating electromagnetic field. This light source paves the way to extremely nonlinear $\mathrm{THz}$ optics and coherent control mediated either by electric or magnetic field coupling to charge and spin degrees of freedom. The specific examples of coherent control of intra-excitonic transitions, collective spin oscillations, non-perturbative nonlinearities in bulk semiconductors and non-adiabatic control of light-matter coupling may illustrate some of the excitement about the emerging field of extremely nonlinear $\mathrm{THz}$ optics: Not only does it open unforeseen vistas in condensed matter physics, but it also facilitates a new kind of $\mathrm{THz}$ quantum optics and sub-cycle quantum electrodynamics.

\section{REFERENCES}

[1] For an overview, see Ferguson, B., and Zhang, X.-C., "Materials for terahertz science and technology", Nature Materials 1, 26 (2002) and M. Tonouchi, "Cutting-edge terahertz technology", Nature Photonics 1, 97 (2007).

[2] Liu, K., Xu, J., and Zhang, X.-C., "GaSe crystals for broadband terahertz wave detection", Appl. Phys. Lett. 85, $863(2004)$.

[3] Kübler, C., Huber, R., Tübel, S., and Leitenstorfer, A., "Ultrabroadband detection of multi-terahertz field transients with GaSe electro-optic sensors: Approaching the near infrared", Appl. Phys. Lett. 85, 3360 (2004).

[4] Zentgraf, T., Huber, R., Nielsen, N., Chemla, D.S., and Kaindl, R.A., "Ultrabroadband 50-130 THz pulses generated via phase-matched difference frequency mixing in $\mathrm{LiIO}_{3}$ ”, Opt. Express 15, 5775 (2007).

[5] Sell, A., Scheu, R., Leitenstorfer, A., and Huber, R., "Field-resolved detection of phase-locked infrared transients from a compact Er:fiber system tunable between 55 and 107 THz", Appl. Phys. Lett. 93, 251107 (2008).

[6] Huber, R., Tauser, F., Brodschelm, A., Bichler, M., Abstreiter, G., and Leitenstorfer, A., "How many-particle interactions develop after ultrafast excitation of an electron-hole plasma", Nature 414, 286 (2001); Huber, R., Kübler, C., Tübel, S., Leitenstorfer, A., Vu, Q.T., Haug, H., Köhler, F., and Amamm, M.-C., "Femtosecond Formation of Coupled Phonon-Plasmon Modes in InP: Ultrabroadband THz Experiment and Quantum Kinetic Theory", Phys. Rev. Lett. 94, 0274011 (2005).

[7] Kübler, C., Ehrke, H., Huber, R., Lopez, R., Halabica, A., Haglund Jr., R.F., and Leitenstorfer, A., "Coherent Structural Dynamics and Electronic Correlations during an Ultrafast Insulator-to-Metal Phase Transition in $\mathrm{VO}_{2}$ ", Phys. Rev. Lett. 99, 116401 (2007).

[8] Kaindl, R.A., Carnahan, M.A., Hägele, D., Lövenich, R., and Chemla, D.S., "Ultrafast terahertz probes of transient conducting and insulating phases in an electron-hole gas", Nature 423, 734 (2003).

[9] Kampfrath, T., Perfetti, L., Schnapper, F., Frischkorn, C., and Wolf, M., "Strongly Coupled Optical Phonons in the Ultrafast Dynamics of the Electronic Energy and Current Relaxation in Graphite", Phys. Rev. Lett. 95, 187403 (2005).

[10] Kröll, J., Darmo, J., Dhillon, S. S., Marcadet, X., Calligaro, M., Sirtori, C., and Unterrainer, K., "Phase-resolved measurements of stimulated emission in a laser", Nature 449, 698 (2007).

[11] Golde, D., Wagner, M., Stehr, D., Schneider, H., Helm, M., Andrews, A.M., Roch, T., Strasser, G., Kira, M., and Koch, S.W., "Fano Signatures in the Intersubband Terahertz Response of Optically Excited Semiconductor Quantum Wells", Phys. Rev. Lett. 102, 127403 (2009). 
[12] Stepanov, A.G., Kuhl, J., Kozma, I.Z., Riedle, E., Almasi, G., and Hebling, J., "Scaling up the energy of THz pulses created by optical rectification", Opt. Express 13, 5762 (2005).

[13] Löffler, T., Kreß, M., Thomson, M., Hahn, T., Hasegawa, N., and Roskos, H.G., "Comparative performance of terahertz emitters in amplifier-laser-based systems”, Semicond. Sci. Technol. 20, 134 (2005).

[14] Xie, X., Xu, J., Dai, J., and Zhang, X.-C., "Enhancement of terahertz wave generation from laser induced plasma", Appl. Phys. Lett. 90, 141104 (2007).

[15] Bartel, T., Gaal, P., Reimann, K., Woerner, M., and Elsaesser, T., “Generation of single-cycle THz transients with high electric-field amplitudes", Opt. Lett. 30, 2805 (2005).

[16] Sell, A., Leitenstorfer, A., and Huber, R., "Phase-locked generation and field-resolved detection of widely tunable terahertz pulses with amplitudes exceeding $100 \mathrm{MV} / \mathrm{cm}$ ”, Opt. Lett. 33, 2767 (2008).

[17] Baltuska, A., Fuji, T., and Kobayashi, T., "Controlling the Carrier-Envelope Phase of Ultrashort Light Pulses with Optical Parametric Amplifiers”, Phys. Rev. Lett. 88,133901 (2002).

[18] Baum, P., Lochbrunner, S., Piel, J., and Riedle, E., "Phase-coherent generation of tunable visible femtosecond pulses", Opt. Lett. 28, 185 (2003).

[19] Erny, C., Moutzourisss, K., Biegert, J., Kühlke, D., Adler, F., Leitenstorfer, A., and Keller, U., "Mid-infrared difference-frequency generation of ultrashort pulses tunable between 3.2 and $4.8 \mu \mathrm{m}$ from a compact fiber source", Opt. Lett. 32, 1138 (2007).

[20] Adler, F., Sell, A., Sotier, F., Huber, R., and Leitenstorfer, A., “Attosecond relative timing jitter and 13 fs tunable pulses from a two-branch Er:fiber laser", Opt. Lett. 32, 3504 (2007).

[21] Chalus, O., Bates, P.K., Smolarski, M., and Biegert, J., "Mid-IR short-pulse OPCPA with micro-Joule energy at 100 kHz", Opt. Express 17, 3587 (2009).

[22] Sell, A., Krauss, G., Scheu, R., Huber, R., and Leitenstorfer, A., "8-fs pulses from a compact Er:fiber system: quantitative modelling and experimental implementation", Opt. Express 17, 1070 (2009).

[23] Krauss, G., Lohss, S., Hanke, T., Sell, A., Eggert, S., Huber, R. and Leitenstorfer, A., "Synthesis of a single cycle of light with compact Er:fiber technology", Nature Photonics, in press (January 2010).

[24] Leinß, S., Kampfrath, T., Volkmann, K.V., Wolf, M., Steiner, J.T., Kira, M., Koch, S.W., Leitenstorfer, A., and Huber, R., "Terahertz coherent control of optically dark paraexcitons in $\mathrm{Cu}_{2} \mathrm{O}$ ", Phys. Rev. Lett. 101, 246401 (2008).

[25] Kira, M., Koch, S.W., "Many-body correlations and excitonic effects in semiconductor spectroscopy”, Prog. Quant. Electr. 30, 155 (2006).

[26] Kampfrath, T., Sell, A., Klatt, G., Pashkin, A., Mährlein, S., Dekorsy, T., Wolf, M., Fiebig, M., Leitenstorfer, A., and Huber, R., "Ultrafast magnetic twist: How intense terahertz transients control spin waves coherently", submitted for publication (2009).

[27] Dini, D., R. Köhler, A. Tredicucci, G. Biasiol, and L. Sorba, "Microcavity Polariton Splitting of Intersubband Transitions", Phys. Rev. Lett. 90, 116401 (2003).

[28] Anappara, A., Tredicucci, A., Biasiol, G., and Sorba, L., "Electrical control of polariton coupling in intersubband microcavities", Appl. Phys. Lett. 87, 051105 (2005); Anappara, A., De Liberato, S., Tredicucci, A., Ciuti, C., Biasiol, G., Sorba, L., and Beltram F., "Signatures of the ultrastrong light-matter coupling regime", Phys. Rev. B Rapid 79, 201303 (2009).

[29] Todorov, Y., Andrews, A.M., Sagnes, I., Colombelli, R., Klang, P., Strasser, G., and Sirtori, C., "Strong LightMatter Coupling in Subwavelength Metal-Dielectric Microcavities at Terahertz Frequencies", Phys. Rev. Lett. 102, 186402 (2009).

[30] Ciuti, C., Bastard, G., and Carusotto, I., "Quantum vacuum properties of the intersubband cavity polariton field", Phys. Rev. B 72, 115303 (2005); De Liberato, S., Ciuti, C., and Carusotto, I., "Quantum Vacuum Radiation Spectra from a Semiconductor Microcavity with a Time-Modulated Vacuum Rabi Frequency”, Phys. Rev. Lett. 98, 103602 (2007).

[31] Günter, G., Anappara, A.A., Hees, J., Sell, A., Biasiol, G., Sorba, L., De Liberato, S., Ciuti, C., Tredicucci, A., Leitenstorfer, A., and Huber, R., "Sub-cycle switch-on of ultrastrong light-matter interaction," Nature 458, 178 (2009). 